Troubler l'expérience sensorielle. De l'utilisation de la réalité virtuelle en anthropologie

\title{
Hugo Montero
}

\section{(2) OpenEdition \\ Journals}

Édition électronique

URL : http://journals.openedition.org/pa/1116

DOI : 10.4000/pa.1116

ISSN : 2273-0362

Éditeur

Université Lumière Lyon 2

\section{Édition imprimée}

Date de publication : 28 juillet 2020

Pagination : 106-126

ISSN : 1634-7706

\section{Référence électronique}

Hugo Montero, «Troubler l'expérience sensorielle. De l'utilisation de la réalité virtuelle en

anthropologie », Parcours anthropologiques [En ligne], 15 | 2020, mis en ligne le 20 juillet 2020, consulté le 03 septembre 2020. URL : http://journals.openedition.org/pa/1116 ; DOI : https://doi.org/10.4000/ pa. 1116 


\title{
Troubler l'expérience sensorielle.
}

\section{De l'utilisation de la réalité virtuelle en anthropologie}

\author{
Hugo Montero \\ Ecole Nationale des Travaux Publics de l’Etat - ENTPE, LAET
}

\section{INTRODUCTION}

Cet article vise à approcher la manière dont certains outils du numérique modifient le rapport qu'entretiennent les chercheurs et chercheuses au terrain ${ }^{1}$ et aux personnes participant à ce dernier. En réponse à la question posée dans ce numéro sur la possibilité $d^{\prime}$ " une distanciation entre le chercheur et le participant », je souhaite ici questionner la distance en la concevant non pas forcément comme un éloignement, mais comme un intervalle mouvant mettant en relation les chercheurs, le terrain et les participants. L'anthropologie du sensible s'est intéressée très tôt à la question de la distance en raison de la difficulté à approcher son objet, le sensible ${ }^{2}$, comprenant les sens en tant qu' « intermédiaire[s] conceptuel[s] » (Colon, Candau, $2014: 17$ ) et dont le visuel tient le haut du pavé (Pink, 2006 ; Howes, 2005 ; Ingold, 2013) au détriment d'autres sensations dans le discours des participants. La révolution sensorielle annoncée par Michel Serres (1985) et le sensory turn interprété par David Howes (2005) n' ont pas eu lieu sans l'aide d'un autre domaine, lui aussi en développement: les humanités numériques. Proposant une approche transdisciplinaire à l'aide d'outils numériques en sciences humaines et sociales (Dacos, 2011), les humanités numériques ont une nouvelle fois transformé les

\footnotetext{
${ }^{1}$ Le terme de terrain sera utilisé ici pour caractériser l'espace-temps où a lieu « une expérience du partage du sensible» (Laplantine, 2007: 47). Ce terme revêt différentes significations au gré des publications (voir par exemple le volume 15 de la revue Hypothèses à ce propos).

2 Les termes sensible et sensorium sont utilisés pour éviter l'écueil que peut présenter le terme «multi-sensoriel », sous entendant le fait que nos sens pourraient être des entités séparées, fonctionnant indépendamment. David Howes propose de parler d'appareils perceptifs (2005), qui impliqueraient plusieurs sens, entremêlés et sollicités à différents degrés, dans la réalisation d'une action. Néanmoins, cet article porte sur différents media qui eux, fonctionnent en séparant les sens. Je dois donc régulièrement « diviser » la sensorialité à la manière de ces media, pour rendre le propos compréhensible, tout en gardant en tête qu'il n'y a jamais de solipsisme sensoriel.
} 
liens que tissent les chercheurs et les participants sur le terrain. Bien que des transformations liées à l'utilisation de media sur le terrain aient été présentes dès les prémices de l'ethnographie, ne serait-ce qu'avec l'écriture, les outils numériques viennent prolonger ces mutations en permettant notamment une aubaine pour l'anthropologie du sensible - de s'intéresser à des registres du monde social difficiles d'accès, comme par exemple «[...] les catégories qui constituent le sensorium [des participants] et les différences probables qu'il existe entre elles et celles de l'anthropologue. » (Pink, $2006: 47)^{3}$.

Dans un premier temps, je parcourrai des méthodologies qui portent selon moi des indices de ce que produisent les effets de distance avec le terrain, de la part des participants, mais aussi des chercheurs. Quand la distance que les participants ou les chercheurs prennent au regard d'une expérience de terrain devient difficilement interprétable, car changeante - nous verrons plus loin dans l'article que l'immersion ${ }^{4}$ renforce ce sentiment - je considère que l'on peut parler de trouble. Je fais l'hypothèse que ce trouble est à l'origine d'un partage intime et sensible de la part des participants car il les met dans une position réflexive vis-à-vis de leur propre expérience. Cette position réflexive et ce partage intime et sensible sont deux éléments clés d'une anthropologie $\mathrm{du}$ sensible qui pourraient permettre de passer d'une description de l'environnement des participants à une description de ce que leur fait ressentir cet environnement (Delas, 2015). Les méthodologies abordées me permettront, dans un second temps, de retracer ce qui a constitué des éléments concomitants à la recherche d'un trouble sur le terrain grâce aux outils audiovisuels. À partir de ces éléments, je m'intéresserai aux transformations qu'induit l'utilisation d'enregistrements sonores et d'images à $360^{\circ}$ sur le terrain et leur diffusion dans un casque de réalité virtuelle, au travers des premières expérimentations menées lors de ma recherche actuelle. Nous verrons comment un tel dispositif permet de rejouer des sensations en faisant usage de ce que j'ai appelé une immersion incarnée, tout en laissant aux participants l'interprétation de leur propre sensorium ${ }^{5}$. Enfin, nous nous intéresserons aux limites de l'utilisation d'outils numériques, qui elles aussi produisent des rapports de distance dans la triade chercheurs-terrainparticipants.

\footnotetext{
${ }^{3}$ Toutes les citations de livres et articles en langue anglaise ont été traduites par mes soins. 4 Par immersion, j'entends ici une expérience qui projette temporairement les chercheurs ou les participants dans un espace-temps différent de celui qui les entoure. Cette expérience créée une forme de déterritorialisation. Il s'agirait par exemple du moment où dans la salle obscure d'un cinéma nous omettons ce qui se trouve autour de l'écran pour nous consacrer pleinement à ce qui est projeté sur ce dernier.

5 Le terme sensorium est ici utilisé en suivant les travaux de McLuhan et de Ong, qui le définissent comme " the entire sensory apparatus as an operational complex " (Ong, $1967: 6$ ). Il ne s'agit pas d'un organe mais d'un ensemble de sensations qui varient d'un individu à l'autre, d'une société à une autre (Howes, 1990 : 99).
} 


\section{ENCHEVÊTREMENTS}

\section{Media \& terrain(s)}

Lorsque Bronislaw Malinowski prit le chemin du terrain - de manière quelque peu forcée - durant la première guerre mondiale pour tracer la route de ce que l'on appela dès lors « l'observation participante », il tenait à jour - en bon ethnographe - des carnets (Malinowski, 1985 [1967]). Il y notait ce qu'il apprenait chaque jour, mais aussi ses impressions, ses observations et ses pensées sur l'expérience qu'il était en train de vivre à des milliers de kilomètres de sa ville natale.

$\mathrm{Au}$ travers de cette écriture, dite ethnographique, le chercheur porte un regard sur un espace-temps qu'il fréquente et arpente. Cette écriture, comme peuvent le faire d'autres types d'écriture, pose une distance réflexive sur l'objet de ce regard. Elle ne peut pas matériellement se faire dans l'immédiateté de l'expérience, et est donc empreinte d'une certaine distance, à minima spatio-temporelle. Cette distance dépend fortement de celui ou celle qui écrit, et varie d'un chercheur ou d'une chercheuse à l'autre. Certaines personnes, ne souhaitant pas apparaître là où elles travaillent munies d'un carnet, préfèrent notifier, classer et documenter ce qu'elles ont appris et échangé à la fin de la journée. $\mathrm{D}^{\prime}$ autres choisissent d'assumer cette position en notant tout ce qu'ils partagent avec les autres directement sur place. Bref, dans ces différents cas la distance est bien là, elle est l'intervalle qui conditionne l'aller-retour entre ce dont nous faisons l'expérience, en tant que chercheurs, et ce que nous écrivons à propos de cette dernière. Robert Desjarlais (2003) va au-delà de cette idée, en comparant ces deux moments - l'in-situ et la réflexivité - aux deux brins d'une corde tressée, formant à eux deux ce que nous appelons plus globalement l'expérience.

Je souhaite justement développer dans cette partie le jeu de distance que nous a permis l'utilisation d'outils comme ceux du numérique, dans le rapport au terrain et même plus largement dans la recherche. Plus que de gommer ou d'effacer cette distance, nous changeons les rapports et les échelles que comporte le terrain, non seulement lors du déroulement de celui-ci, mais aussi dans notre bureau, ou notre lieu de travail. Ce regard posé sur ces transformations ne se veut ni exhaustif ni chronologique. Il est important de garder à l'esprit que l'utilisation de différents media sur le terrain résulte souvent d'une combinaison voir de la progression d'une recherche ou $\mathrm{du}$ temps passé sur place.

\section{Capturer l'instant}

La photographie a été utilisée avant 1920 comme moyen de documenter et de donner à voir ce qui a eu lieu sur des terrains éloignés ou difficiles d'accès ainsi que pour « explorer la société » (Becker, $1974: 3$ ). Concernant la question de la distance qu'impose l'utilisation d'un tel medium, la photographie 
semble sur de nombreux points très différente des effets produits par l'écriture. Lorsque l'écriture se situe nécessairement après l'action - elle en décrit ses lignes à partir de souvenirs ou d'autres matériaux -, la photographie, elle, est le medium de l'instant : elle capture un moment précis, une image d'une action. Eadweard Muybridge (1901) l'avait bien saisi en envisageant de comprendre ce qui fait mouvement à partir de multiples photographies. Dès lors, et surtout quand elle s'éloigne de la nécessité de poses longues, la photographie devient une alliée de choix pour l'ethnographe. De la même manière que ses carnets, il peut ramener ces images à son bureau, les étudier et les regarder autant de fois qu'il le souhaitera. Contrairement à l'écriture, la photographie de terrain ne permet pas la même prise de distance, c'est-à-dire que l'aller-retour entre ce que l'on souhaite "capturer", par l'écrit ou en appuyant sur le déclencheur d'un appareil photo, n'a pas la même durée et ne s'attarde donc pas sur les mêmes indices. Une première trace de la sérendipité engendrée par le medium apparaît.

Dans un même temps, un autre medium s'est développé, le film qui, bien que n'en étant qu'à ses prémices, a connu une adoption fulgurante et notamment dans le cadre de la recherche, ou du moins de l'exploration. Le film permet de faire l'expérience du mouvement, et avec lui se déploie un certain partage de l'action entre celui qui regarde et celui qui filme. C'est-àdire que le nombre d'images capturées laisse une forte place - notamment dans le mode documentaire - à la sérendipité, à l'inconnu et au hors champ alors si bien assimilé par la photographie. S'autoriser l'aléa de ce qui est hors champ, mêler l'action que l'on souhaite capturer avec les mouvements de la caméra (et cela simultanément) c'est offrir à voir d'autres couches du réel en train de se faire.

\section{Paradigmes et développements technologiques}

Tout comme la photographie, le film a connu les cadres d'un scientisme débridé, qui en firent l'outil par excellence pour capturer de manière exhaustive tout ce qui se déroule dans l'action ${ }^{6}$. Mais, peu de temps après leurs premières utilisations comme matériaux de recherche $"$ objectifs ${ }^{7}$ devant utiliser des objectifs larges, sur trépied, pour ne pas « altérer » l'action (Mead et Bateson, 2002 [1977] : 78), ces deux medium partagèrent l'avènement $\mathrm{du}$ "perspectivisme », c'est-à-dire le fait de considérer que tout savoir (ce qui inclut sa production) est incarné et émane donc d'un point de vue, d'une position dans le monde. Ce tournant a permis de repenser la place $\mathrm{du}$ chercheur en tant que seul «collecteur» de données sur le terrain, et ainsi d'amorcer un autre mouvement dit " écologique » rendant le perspectivisme horizontal, et non plus d'un percevant à un perçu. C'est-à-dire que le travail

\footnotetext{
${ }^{6}$ On peut citer les films réalisés par l'un des précurseurs du cinématographe, Étienne-Jules Marey.

7 Voir l'amusant film Kitchen Stories (2003) pour en saisir les enjeux sous les traits de la fiction.
} 
du regard ne se limite pas à comprendre ce qu'il se passe entre celui qui observe et celui qui est observé (et vice-versa), mais plutôt dans la relation dans son ensemble - ou du moins dans ce que nous en percevons. Le travail du chercheur, qu'il soit écrit, photographique ou filmique, procède alors d'un «fluid authorship » (Grimshaw et Ravetz, 2009). Celui qui décrit l'action, quel que soit le medium, décrit un réel dont il fait partie et qu'il modifie au même titre que ceux qui performent telle ou telle action. Ainsi, «La caméra n'est pas l'équivalent du stylo qui aurait enregistré ce que le cinéaste aurait directement observé, au contraire elle serait un des éléments qui met les corps en action et en relation » (Lallier, $2011:$ 106).

La prise de conscience de la subjectivité de tout regard porté sur un objet, ainsi que de son imbrication dans des relations complexes, a permis le développement de principes tels que la recherche participative, où la production $\mathrm{du}$ travail de recherche implique les participants. Ce principe a débuté dans les milieux militants des années 1960 avec le travail du chercheur et activiste Don Snowden qui proposait aux personnes de filmer leur quotidien et leurs luttes avec lui ; il s'agissait de leur «donner la voix » en les impliquant directement dans le processus de production ( $\mathrm{O}^{\prime}$ Brien et al., 2014 : $1)$.

Dans les années 1990, ce type d'approches connut un succès plus large dans la recherche, notamment en raison de la simplification des techniques de prise de vue et de l'abaissement de leur coût. Une idée simple, mais impensable avant cela, fit son apparition : donner aux personnes avec qui l'on travaille les outils de la captation. L'un des dispositifs développés, nommé photo novella (Wang et Burris, 1994) puis photovoice (Wang et Burris, 1997), est le fait de donner des appareils photo jetables aux participants d'une étude. Le but étant de «donner l'opportunité aux participants de tisser un récit à propos de leurs quotidiens » (idem : 179). Ces photographies seront ensuite commentées par les participants, de manière individuelle ou collective (avec la méthode du focus group), pour faire émerger ce qui dans l'utilisation de ce medium fait sens pour les participants. Cette méthodologie ambitionne d'accéder à un point de vue à la première personne, et d'inviter le chercheur dans des moments intimes du quotidien où la distance permise par le medium abolit certaines barrières, comme la gêne. De plus, elle permet aux participants d'être des acteurs de la recherche, en mettant eux-mêmes la focale, au sens propre, sur un point, un détail, un moment fort pour eux.

Dans le cas du film, la procédure se révèle être bien plus complexe à mettre en place, et c'est réellement l'arrivée de la vidéo qui permettra de changer la donne (on notera d'ailleurs que l'on retrouve bien souvent le terme «videovoice» plutôt que «filmvoice » dans les publications à ce propos). Avoir recours à la vidéo c'est permettre à deux voies d'émerger.

La première voie est directement liée à ce qui a été décrit plus haut concernant le film : le nombre d'images captées pendant une séquence laisse une place importante à la sérendipité : celui qui filme peut se retrouver à 
l'aube d'une rencontre imprévue alors même que la caméra tourne. Cette voie résonne particulièrement avec le type de matériaux filmiques délivrés par les participants. Il s'agit bien souvent de nombreuses heures de rushes dans lesquelles le commencement et l'arrêt de l'enregistrement peuvent être surprenants pour le chercheur, mais qui s'expliquent par la manière dont l'action se déroule hors champ. Le commentaire des participants viendra donc re-contextualiser ce qui a été filmé et lui donner un sens, une direction.

La seconde voie que permet le videovoice est d'inviter le son dans la description de l'action $^{8}$. La combinaison du son et de l'image permet l'activation $d^{\prime}$ ' impressions secondaires» (MacDougall, 2006), d' « effets synesthésiques » (Mohl, $2015: 48)$ parmi lesquels l'on retrouve la question du mouvement, des textures, de $1^{\prime}$ haptique ${ }^{9}$, de la proprioception ${ }^{10}$, des odeurs et des goûts: " nous sentons l'odeur de ce que nous entendons et pouvons voir la dureté et la souplesse des matières » (idem). C'est-à-dire que comme pour le cinéma ${ }^{11}$, les sons et images projetés aux participants ne concernent pas seulement la vue et l'ouïe, mais activent d'autres répertoires sensoriels qui eux-mêmes pourront engendrer des retours réflexifs, comme un récit mémoriel.

Revenons à la question de la distance, qu'évoque directement la manière dont est perçue et vécue l'action par les spectateurs-participants. L'intimité sensorielle que crée la combinaison images-sons décrite précédemment est fortement dépendante d'un autre paramètre : l'immersion. L'immersion, c'està-dire le fait de se fondre, d'être plongé dans une expérience, est à nouveau un rapport de distance. Elle n'est pas limitée au medium vidéo, mais c'est bien dans celui-ci que s'est affinée la recherche d'une telle distance. Le cinéma nous invite dans une salle obscure, où la présence des images et des sons est mise au premier plan grâce à l'agencement de l'espace-temps de la salle. En tant que chercheurs et chercheuses nous devrions peut-être nous inspirer, et

\footnotetext{
${ }^{8}$ Je reviendrai dans la partie suivante sur la question de l'écoute seule du son, que ce soit du « paysage sonore » (Schafer, 1979) ou de «l'environnement sonore » (Battesti, 2013). Ici je me concentrerai sur ce que produit l'alliance images-sons - que nous pouvons appeler medium vidéo dans le but de simplifier la démarche - lors du visionnage d'une action en s'inspirant des recherches menées sur l'expérience cinématographique.

9 James J. Gibson défini l'haptique comme «[...] un dispositif grâce auquel l'individu obtient des informations à la fois sur le milieu et sur son corps. Il sent un objet par rapport au corps et son corps par rapport à un objet. C'est le système perceptif par lequel les animaux et les hommes [sic.] sont littéralement en contact avec l'environnement » (2001 [1966] : 98)

$10 \mathrm{La}$ proprioception est la «perception qu'a l'homme [sic.] de son propre corps, par les sensations kinesthésiques et posturales en relation avec la situation du corps par rapport à l'intensité de l'attraction terrestre » CNRTL, https://www.cnrtl.fr/definition/proprioception 11 Le pouvoir de suggestion porté par le medium cinématographique me semble clairement palpable au travers du documentaire expérimental Leviathan (2012) réalisé par Lucien Castaing-Taylor et Verena Paravel, tous deux membres du Sensory Ethnography Lab. La manière dont ce documentaire est filmé, notamment en utilisant des caméra GoPro attachées au bout de perches suivant à la fois le mouvement du bateau et des vagues, est déroutant. Il laisse le spectateur submergé par des sensations haptiques et optiques.
} 
comprendre, l'attention qui est portée à un tel dispositif (la salle de cinéma) pour pouvoir anticiper et imaginer l'influence des conditions $\mathrm{d}^{\prime}$ «audiovisionnage » des séquences données à voir aux participants. En effet, il me semble crucial, pour viser une expérience qui ne se limite pas à ce que l'on voit et à ce que l'on entend - c'est-à-dire favorable à l'émergence «d'effets synesthésiques »- de créer les conditions idoines à cette expérience ${ }^{12}$. Il est nécessaire ici de souligner un élément souvent dommageable dans nos dispositifs de retransmission : le son. Bien souvent, il est le parent pauvre de nos installations, et bien que disposant d'un puissant vidéoprojecteur, nous en oublions l'emprise, et son impact sur l'expérience $\mathrm{qu}^{\prime}$ en font les participants.

\section{Faire entendre le terrain}

Après avoir abordé la question de la distance en regard de l'écriture ethnographique, de la photographie, du film et de la vidéo, penchons-nous sur les enregistrements sonores en tant que medium autonome car, en plus d'avoir un fonctionnement propre, leur utilisation fait bien souvent partie des options à notre disposition dans nos laboratoires de recherche.

Les enregistrements de terrain se sont d'abord cantonnés à capter une source spécifique, en regard de l'écriture: la musique et/ou les paroles des participants de la recherche. Cela nous indique à quel point l'enregistrement sonore a servi dans un premier temps à préciser le texte. L'écriture ethnographique se concentrant sur les rites et les discours, celle-ci s'accommoda facilement d'un outil pour enregistrer ce qui s'y déroule, en direct.

De la même manière qu'au sujet des media précédemment cités, les paradigmes de la recherche se transforment pour voir l'émergence de ce que l'on nomme la pensée écologique. La recherche acoustique traduira cela par "l'écologie acoustique", qui s'intéresse aux relations en jeu dans un environnement sonore. En 1977, lorsque le compositeur R. Murray Schafer propose le concept de paysage sonore (Schafer, 1979), une petite révolution se met en marche. Il provoque, notamment dans le milieu de la recherche, une prise de conscience : il est possible d'interpréter un environnement sonore à la manière d'un paysage. C'est-à-dire que les sources sont à la fois perceptibles d'un point d'écoute propre et qu'elles s'influencent les unes les autres, créant par cette occasion un phénomène unique. Ce livre, comme bien souvent, fut le résultat d'un processus lancé bien en amont qui vint formaliser un concept déjà dans «l'air du temps ». Le laboratoire du CRESSON13, fondé en 1979 - la même année que la parution de l'ouvrage de Schafer en français -, décide

\footnotetext{
${ }^{12}$ Je reviendrai sur ce point dans un second temps de l'article, pour justifier l'utilisation du casque de « réalité virtuelle » dans un tel processus.

${ }^{13}$ Le Centre de Recherche sur l'Espace Sonore et l'environnement urbain (CRESSON) est de nos jours une équipe de recherche du laboratoire AAU (Ambiances, Architectures, Urbanités UMR 1563).
} 
justement de porter l'oreille à la condition sonore en milieu urbain et va donc développer toute une batterie de méthodologies pour " travailler en son ».

Je souhaite questionner les rapports de distance qu'entretiennent chercheurs et participants en m'appuyant sur deux méthodologies de recherche : l'écoute réactivée ${ }^{14}$ et Mics in the Ears ${ }^{15}$. Parmi les points communs que l'on retrouve entre ces démarches, j'insisterai sur ceux qui jouent de la distance et du trouble chez les participants. En effet, le fait de donner à entendre, et seulement entendre un environnement sonore différent de celui où les participants se trouvent est déjà une première source de trouble. Comme le souligne bien Vincent Battesti au sujet de ses travaux au Caire,

«entendre ou écouter n'est pas "naturel". Entendre, ce n'est pas seulement posséder des oreilles, $c^{\prime}$ est organiser et comprendre, dans un exercice conscient ou non, ce qu'un phénomène mécanique dans notre environnement transmet d'informations à l'oreille et de là, au cerveau. Cette écoute se fait toujours avec des appareils qui ne sont jamais "neutres", et au premier plan, notre oreille. » (Battesti, $2013: 74)$

Dès lors, l'invitation des chercheurs faite aux participants d'écouter un «paysage sonore» ou un "environnement sonore" constitue un premier trouble. Il s'agit d'une expérience peu commune dans le quotidien des participants, qui va les plonger dans une focalisation sur l'écoute. À partir de là, deux rapports de distance se construisent : dans le cas de l'écoute réactivée, les sons sont captés et agencés par les chercheurs. Les participants seront alors en recherche de similarités avec d'autres environnements sonores entendus ainsi que d'indices sonores pouvant leur permettre de situer l'action. Dans le cas de la seconde méthode - Mics in the Ears - les participants ont eux-mêmes capté ce son et il n'y a pas de montage de ce dernier. Ils vont alors rechercher plus précisément dans leur mémoire les espaces traversés et suivant une chronologie, pour pouvoir commenter ce qu'il se passait dans l'action. Dans le premier cas, les participants objectivent et cherchent à trouver ce qui serait commun à cette écoute ainsi qu'à d'autres sonorités entendues dans le passé. Pour le second cas, il s'agit d'identifier les singularités de l'espace-temps pour être à même de commenter précisément, et à la première personne, l'action. Le commentaire renvoie donc à une situation particulière: "Ce qui rend ces enregistrements uniques et personnels est le fait qu'ils ne varient pas seulement dans le temps et l'espace, mais aussi en raison du comportement de chaque participant dans l'espace public. » (Battesti et Puig, $2016: 303$ ).

\footnotetext{
${ }^{14}$ L'écoute réactivée est une méthodologie pensée par Jean-François Augoyard, qu'il définit ainsi : « il s'agit de recueillir les réactions d'habitants ou d'usagers à qui l'on fait entendre les sons de leur propre environnement » $(2001: 128)$.

15 Mics in the Ears est une méthodologie théorisée par Vincent Battesti et Nicolas Puig. Elle invite des habitants à déambuler seuls ou accompagnés dans un lieu qu'ils côtoient régulièrement, équipés de microphones binauraux enregistrant le son des lieux à 360 degrés. Ils sont ensuite invités à ré-écouter ces sons en les commentant (Battesti et Puig, 2016).
} 
Une autre différence notable entre ces deux méthodologies et leurs effets sur la distance vécue par les participants est l'utilisation de différents types de microphones. La seconde méthode, innove, en ce sens qu'elle propose l'usage d'un dispositif léger et mobile qui enregistre un son à 360 degrés, dit binaural ${ }^{16}$. Il permet de restituer lors de son écoute un son spatialisé, c'est-àdire prenant en compte précisément la place de la source dans l'espace au moment de l'enregistrement. Les microphones utilisés de nos jours (et dans la seconde méthodologie présentée) sont composés de deux cellules omnidirectionnelles portées dans les oreilles, qui recréent donc les conditions d'écoute à partir de nos oreilles. Les participants témoignent « d'une sensation d'être dans la même situation, d'être exposés aux mêmes sons que ceux perçus pendant la marche. » (Battesti et Puig, 2016 : 303).

L'écoute d'un tel type d'enregistrements est une expérience forte - d'autant plus dans la multitude de sons que propose l'espace urbain. Elle plonge le participant dans un univers sonore à explorer. Il tente de situer cet espacetemps, ce qui trouble - du moins au début - sa perception et l'engage dans un questionnement en vue de l'identification de chaque source d'émission. De plus - et là il s'agit d'un point commun aux deux méthodologies - l'expérience d'un enregistrement sonore est tout à fait différente en termes d'engagement ; elle propose d'écouter un espace-temps plutôt que de le voir: "l'espace sonore est différent de l'espace lumineux : contrairement à la lumière, le son nous atteint de toute part, voyageant d'autres pièces, rues, espaces, jusqu'à nos oreilles. » (idem)

J'ai ici retracé la question de la distance, dans les paradigmes de la recherche, mais aussi au travers de l'évolution, de l'accès et de la miniaturisation de certaines technologies dorénavant "outils de recherche ». Elle pose les bases à la construction d'autres méthodologies tirant profit de ces enseignements et voulant développer les «jeux de distance » permis par l'utilisation d'autres media. La captation, mais aussi la réception de ce type d'expérimentations sont dépendantes de la précision des outils utilisés, ainsi que des conditions dans lesquelles elles sont restituées. Il m'a alors paru important, dans la construction méthodologique qui va suivre, d'insister sur ces deux points en tentant de se rapprocher de ce que permet l'ethnographie expérimentale ${ }^{17}$. Le glissement vers l'utilisation d'un casque de "réalité virtuelle » s'est donc fait à partir de ces éléments et avec l'idée de reposer un

\footnotetext{
${ }^{16}$ C'est un procédé découvert en 1839 et resté à l'état de curiosité jusqu'à sa théorisation en 1973 par Gérald Oster.

17 Le développement de l'ethnographie expérimentale par des institutions comme le Sensory Ethnography Lab. ou bien des réalisateurs comme Ben Rivers et Ben Russel permet de mélanger les évocations du terrain et les gestes artistiques des auteurs-chercheurs, en jouant sur le medium cinématographique pour obtenir un entre deux intéressant et déroutant. Ce travail, qui reste encore en devenir, nous invite à «désamorcer ces fameux 'allochronismes' (Fabian, 1983) » (Accolas, 2015 : 311). Ce décalage, qui malgré le fait que le terrain est un espace-temps partagé avec les participants, reste peu interprété comme tel par les chercheurs.
} 
certain nombre d'acquis concernant la distance que les chercheurs comme les participants négocient avec le terrain.

\section{UN DÉVELOPPEMENT : L’EXPÉRIENCE RÉACTIVÉE}

Poursuivant une réflexion engagée par l'anthropologie du sensible, ma recherche actuelle vise à expliciter la dimension sensible de marches quotidiennes dans l'espace urbain. Avec la préoccupation de recueillir un discours sensible des habitants à propos de leurs pratiques, je me suis dirigé vers un retour à la première personne. Cette méthodologie, que j'ai appelé l'expérience réactivée, $s$ inspire notamment d'autres méthodologies comme l'écoute réactivée, videovoice et Mics in the ears, et je reviendrai sur leurs implications dans son déroulé.

\section{Marche équipée}

L'une des premières étapes de cette méthodologie ${ }^{18}$ consiste à proposer aux participants (en l'occurrence les habitants d'un même quartier) de s'équiper $\mathrm{d}^{\prime}$ un dispositif de captation lors de l'un de leurs trajets quotidiens et à pied ${ }^{19}$. Comme dans la méthodologie Mics in the ears, ce dispositif est composé d'une paire d'écouteurs intraauriculaires disposant de microphones omnidirectionnels et permettant d'enregistrer un son binaural en stéréo. Cette partie audio est couplée avec une caméra enregistrant l'image à 360 degrés à l'aide de deux objectifs, l'un de chaque côté de la caméra. Cette dernière est disposée au niveau de l'épaule des participants, sur l'anse d'un sac à dos (Figure 1).

\footnotetext{
${ }^{18}$ Cette méthodologie a été imaginée en collaboration avec Denis Cerclet, anthropologue au laboratoire EVS (UMR 5600).

19 Dans les deux exemples utilisés pour cet article il s'agit d'un trajet domicile-travail et dans l'autre cas domicile-loisir - pour rejoindre la piscine municipale du quartier.
} 


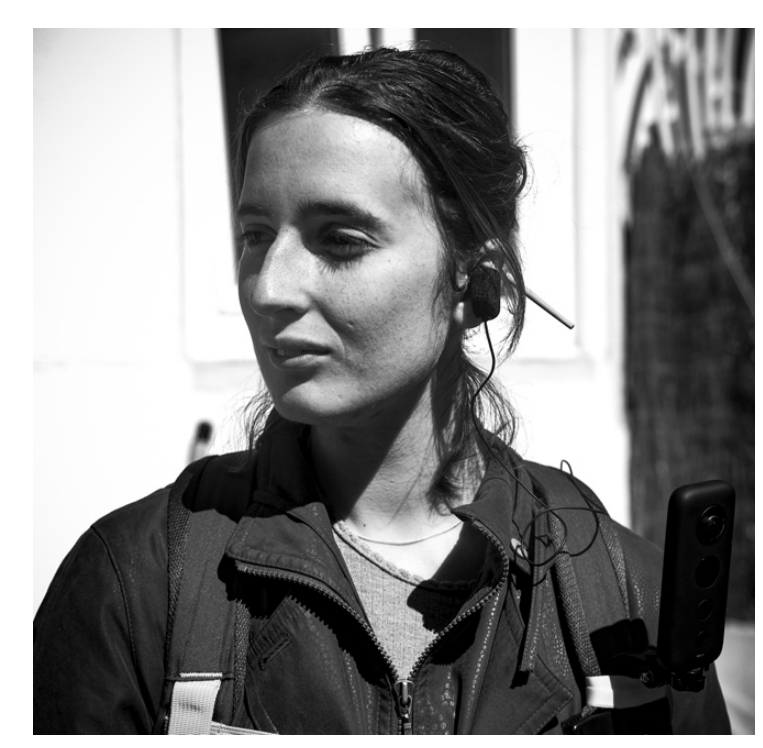

Figure 1 : Une participante portant le dispositif. Crédits : Hugo Montero

Cette position a été choisie pour permettre à la caméra de filmer derrière la personne ainsi que d'un point de vue proche de la tête, mais moins mouvementé que cette dernière.

\section{Dessiner, écouter, commenter}

Une fois cette marche équipée terminée, les participants sont invités, plus tard dans la journée, à revenir sur ce moment. Au travers de différentes étapes, ils vont se remémorer leur trajet. Dans le cas présent, il leur est proposé de dessiner une carte mentale de leur parcours et d'écouter les traces sonores de ce dernier pour ensuite commenter leur expérience. Il est nécessaire de préciser que le temps qui sépare cette marche de leur retour est non seulement dû au temps que peuvent consacrer les participants à cette expérimentation, mais aussi aux contraintes techniques, obligeant à prévoir un temps long de traitement des données entre les enregistrements et leur implémentation dans le casque de réalité virtuelle. Ces étapes permettent aux participants de se repasser, à l'aide de différents media, le trajet effectué plus tôt.

Les cartes mentales peuvent être représentées de différentes manières, laissées à la discrétion des participants : le trajet peut être illustré par des traits, des couleurs, des flèches. Certains participants font le choix de dessiner directement des rues, d'autres de détailler certains points et d'en légender $\mathrm{d}$ 'autres. Cette étape permet d'explorer une première relation de distance avec l'expérience. Les participants choisissent un point de vue (déjà insinué par l'utilisation du terme carte mentale) différent de celui adopté durant la marche. Ils s'éloignent des rues pour représenter leur cheminement, dans les deux cas illustrés ici, vu du dessus (Figures 2 et 3). Ce point de vue est inédit 
pour les participants, mais aussi pour le chercheur, qui voit apparaître au fur et à mesure du dessin une manière de représenter un espace-temps donné.
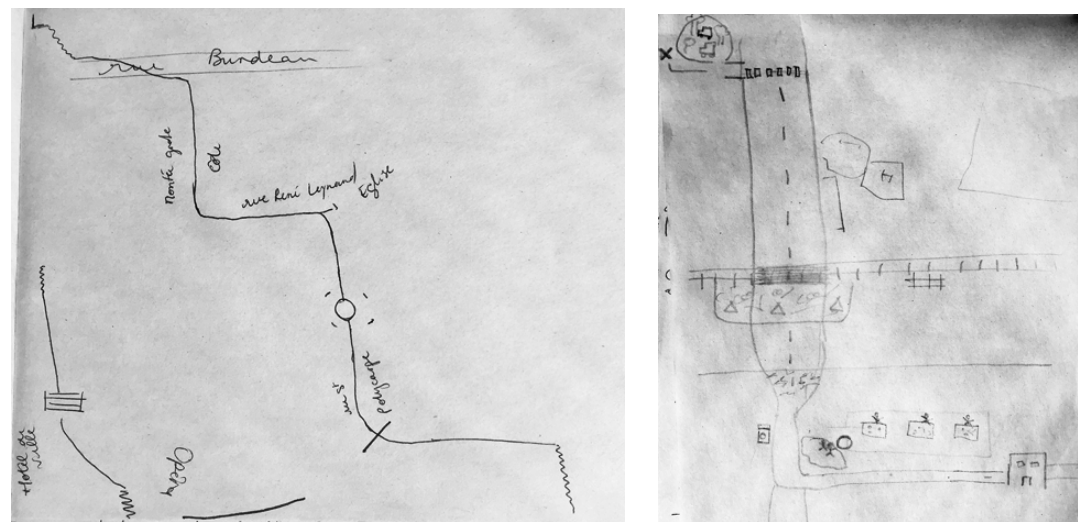

Figures 2 et 3 : Deux cartes mentales réalisées par les participantes. Crédits : Hugo Montero

L'écoute quant à elle permet aux participants d'entendre le son spatialisé qu'ils ont capté durant leur trajet. Cette première écoute a notamment pour but de les faire entrer dans un moment hautement réflexif à propos de leur trajet, de chercher le sens des signaux sonores entendus et de se (re)situer dans cet espace-temps. Cette étape se révèle réflexive pour les participants en raison de la difficulté à s'habituer à ce type d'écoute : « les participants peuvent être gênés dans un premier temps : l'écoute au travers d'un casque stéréo est une compétence qui s'acquiert, et non universelle, néanmoins, cette difficulté n'est pas insurmontable » (Battesti et Puig, 2016 : 304). De plus l'aspect immersif de ce type d'enregistrements invite les participants à se plonger dans l'espacetemps perçu, d'autant plus engageant que la captation est faite en mouvement. Enfin, l'intervention du chercheur pour poser des questions brèves comme «où êtes-vous ? » permet de lancer ou relancer les questionnements des participants vis-à-vis de leur position et de leurs sensations.

Écouter l'enregistrement sonore d'un trajet induit une relation à l'environnement peu exploitée dans notre quotidien. C'est-à-dire que nous ne parcourons habituellement pas un tel trajet à l'oreille uniquement. Pour naviguer dans cet espace, nous allons partir à la recherche d'éléments marquants, d'évènements sonores de la marche permettant de faire le lien avec nos souvenirs de ces moments. Comme le dit Julien Delas à propos d'une expérimentation sonore: "Les parcourants ne peuvent plus anticiper leur environnement sans leur vue (ou alors de façon très limitée) et se retrouvent à faire constamment l'épreuve de surgissements. »(Delas, 2015 : 58). Comme en témoigne la réaction d'une participante lors d'un entretien : "Ah ouais là on commence à entendre le bruit, c'est là qu'il y avait les travaux... c'était assez impressionnant. ».

Concentrés, le regard fixe ou les yeux fermés les participants recherchent intensément à donner du sens à ce dont ils font l'expérience, à relier les sensations sonores aux sources précédemment vues et entendues. Il s'agit 
pour eux de faire face à une version sonore de ce que Robert Bonamy nomme le fond cinématographique: "L'hypothèse du fond cinématographique s'oppose à une localisation précise et stable dans un espace, elle implique d'identifier un milieu dans lequel les personnages s'inscrivent et agissent : le milieu ambiant, le plus souvent l'air, occupé aussi de sonorités, dans lequel les figures humaines et les objets prennent place.» (2013: 22) Ainsi les participants se plongent dans une enquête sonore retraçant les ambiances des lieux parcourus.

En abordant de nouvelles manières de faire l'expérience d'un espace-temps connu, c'est bien la distance avec la première expérience (le trajet effectué pendant la marche équipée) qui est en question, pour les participants qui revisitent un trajet quotidien - posant dessus d'autres regards / écoutes, tout comme pour le chercheur. En effet, ce dernier partage avec les participants leurs manières de représenter graphiquement et verbalement une expérience donnée. D'un côté, les participants dessinent et donc représentent à leur manière leur trajet - avec pour limites le cadre posé par les chercheurs -, de l'autre ils donnent du sens à un enregistrement sonore encore peu signifiant : "alors que les microphones enregistrent tout, incluant le fond sonore et d'autres sont plus proéminents, le couple cerveau-oreille est capable de filtrer les signaux entrants. C'est pour cela que l'interprétation des participants est cruciale. » (Battesti et Puig, 2016 : 303).

\section{Mettre le casque de réalité virtuelle}

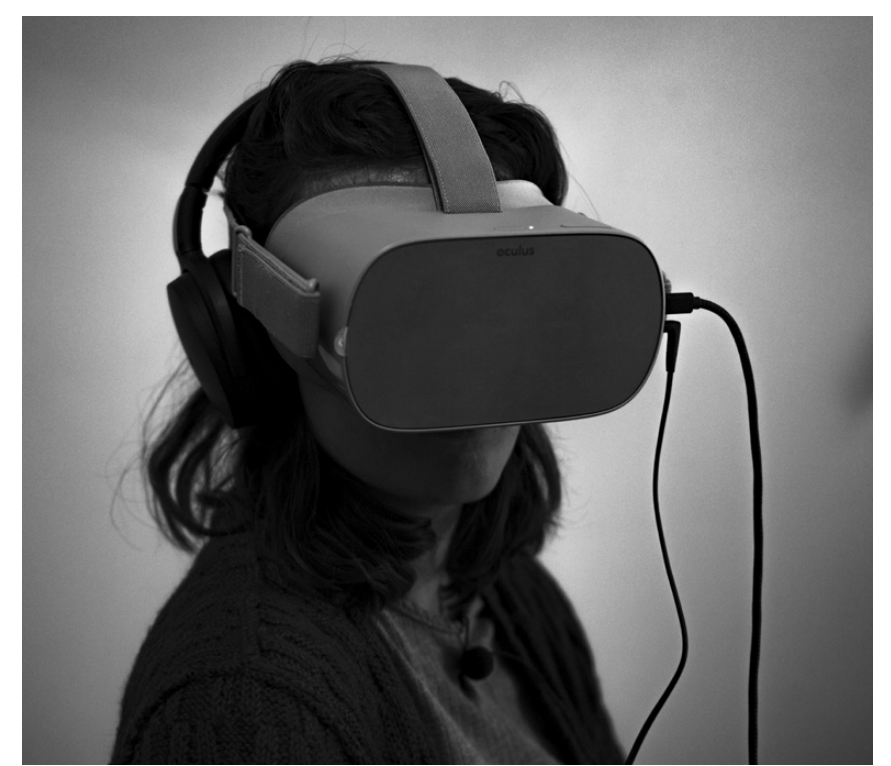

Figure 4 : Une participante faisant l'expérience de sa marche via un casque de réalité virtuelle (VR). Crédits : Hugo Montero

«Les questions posées par la photographie et le cinéma touchet, sans doute, à ce qu'il y a de plus étrange et inquiétant dans notre expérience : l'oscillation entre le voir et ne pas tout voir, le croire et le ne pas croire. Elles concernent la production, 
la diffusion et la réception des images, dans leurs rapports problématiques au réel. » (Laplantine, 2007 : 50)

Dans cette citation, François Laplantine met en avant la manière dont deux media, la photographie et le cinéma, modifient notre rapport au réel. Deux points nodaux de notre expérience, et leurs antagonismes, sont rejoués par ces media : les couples voir/ne pas tout voir et croire/ne pas croire. Je pense que l'écoute d'un enregistrement sonore, notamment lorsqu'il est spatialisé, touche en partie les mêmes « rapports problématiques au réel ». C'est-à-dire que lors d'une telle écoute, les participants oscillent entre l'entendre et ne pas tout entendre, ainsi qu'entre le croire et le ne pas (tout) croire.

Ce que j'appellerai le trouble - regroupant ici « ce qu'il y a de plus étrange et inquiétant » (Laplantine, 2007: 50) - est d'autant plus déroutant pour les participants lorsqu'ils mettent un casque de réalité virtuelle dans lequel est projeté une image à 360 degrés ainsi qu'un casque audio recréant un son à 360 degrés. Cette combinaison a la particularité de permettre une immersion incarnée, c'est-à-dire de plonger directement le spectateur dans une expérience dont la distance est difficile à interpréter. Contrairement au dispositif de la salle de cinéma, dont la fenêtre qu'est l'écran pose une distance facilement interprétable (on peut échapper à l'action en regardant ailleurs par exemple), la VR et le son binaural sont quant à eux englobants, mais aussi envahissants. Dire d'une telle immersion qu'elle est "incarnée » est une tentative pour souligner le fait que les mouvements de notre tête sont fortement liés à notre expérience visuelle lors d'une telle expérience. Bien que ces derniers le soient aussi lorsque l'on regarde, ou pas, un écran, la synchronicité entre les mouvements de notre tête et le déplacement du cadre dans un casque de réalité virtuelle, crée une immersion tout à fait particulière : où que l'on regarde il y a quelque chose à observer, une image en train de se faire. Il s'agit d'une représentation du monde à laquelle nous ne sommes pas du moins pas encore - habitués, d'une manière de représenter l'espace et le temps qui désynchronise deux actions : celle de regarder et celle d'aller vers. Le participant peut alors regarder là où il le souhaite sans se soucier de mettre un pied devant l'autre. Cela peut sembler un principe simple, mais dans le cas d'une telle expérience les participants sont partagés entre deux actions: regarder devant soi et suivre la marche, ou bien explorer les alentours à la manière d'un spectateur de l'action. Dans les deux cas présentés ici, j'ai pu observer une oscillation constante entre ces deux actions, avec une tendance lourde pour la première ; la majeure partie du visionnage des participants se passe avec un regard face à soi, qui suit la direction des pas (Figure 5). 


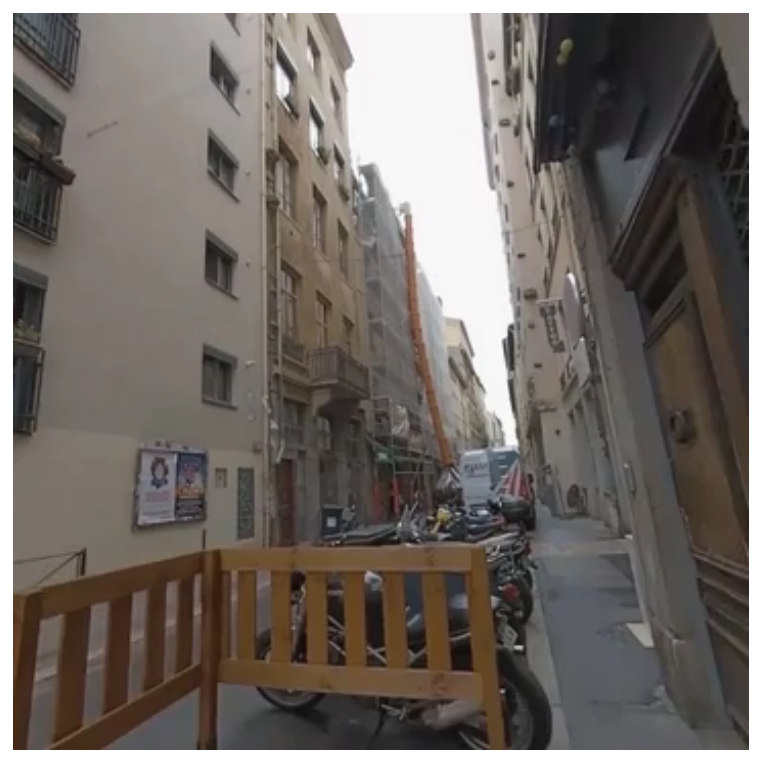

Figure 5 : Vue du casque VR. Une participante passe près d'une importante source sonore. (URL: https://vimeo.com/339848870/d8ea2dbacc) Crédits : Hugo Montero

L'utilisation d'un casque de réalité virtuelle, dans les conditions proposées par cette méthodologie (et que l'on pourrait retrouver dans des expérimentations futures), n'est pas très répandue. J'ai pu constater lors des premiers essais qu'il était nécessaire de passer par une phase d'adaptation au medium pour éviter des maux de cœur ou un stress de la part des participants, comme en témoigne une participante : «C'est vraiment le bas qui est impressionnant [...]». Pour faire en sorte que cette première - parfois deuxième - expérience VR se passe bien, $\mathrm{j}^{\prime}$ ai conçu ${ }^{20}$ ce que j'appelle un sas. Il s'agit d'une vidéo prise en plan fixe et présentant une situation commune. Dans celle-ci, la personne pourra observer autour d'elle, comprendre comment s'utilise une image à 360 degrés et comment elle est projetée dans un casque, tourner la tête et apercevoir quelque chose, entendre un bruit venant de derrière soi et être capable de regarder sa source, en bref s'habituer à une expérience immersive. Cette expérience passe le plus souvent par une période où la personne est crispée et surprise par la forme que prend la réalité virtuelle, et par le nombre important d'informations qui lui parviennent au même moment. Au fur et à mesure, les participants expérimentent le medium et s'offrent des libertés, comme celle de regarder derrière eux ou à leurs pieds. Ce sas leur permet d'aborder sereinement la marche qui va suivre. Néanmoins, un effet persistera durant l'expérience, celui de la dissociation entre le lieu où l'on se trouve, un bureau en l'occurrence, et le lieu où a été filmée la marche. Notre organisme est troublé par cette temporaire ubiquité, qui crée des sensations qu'il sera intéressant d'étudier par la suite.

\footnotetext{
20 Sous les recommandations de Grégoire Cattan, doctorant qui travaille pour l'entreprise IHMTEK en collaboration avec le GIPSA-LAB (UMR 5216) basé à Grenoble.
} 
Pour l'heure, les troubles induits par l'utilisation d'un casque de réalité virtuelle doivent être régulés voire atténués par les chercheurs, pour ne pas remettre en cause la faisabilité d'une telle expérience, notamment en utilisant une caméra stabilisée qui permet de pouvoir mieux suivre l'action pour les participants. Ces troubles restent tout de même des indicateurs importants du rapport de distance qui se joue lors d'une telle expérimentation et de son interprétation changeante de la part des participants. C'est ce qui m'a conduit à formuler l'hypothèse que l'image d'une expérience récente retransmise au travers d'un casque de réalité virtuelle ainsi que le son de celle-ci, spatialisé, seraient à même d'embarquer les participants dans des impressions telles que celle de marcher, de sentir l'odeur du gasoil d'une voiture passant près d'eux, de frôler une personne marchant sur le trottoir ou de sentir la chaleur du soleil sur leur peau. Cela peut se faire par le tissage de la mémoire des lieux et des instants encore récents avec celui de leur présence corporelle à l'espace et au temps $^{21}$. Ce dispositif méthodologique donne une forte place à notre corps, le rendant bien visible lors de la réactivation; le rythme du corps ainsi que ses postures sont des indicateurs de ce qui est perçu et, par mimétisme, ressenti par les participants. Pour prendre un exemple, lors des expérimentations, l'une des participantes s'est écriée, avec le casque sur la tête: «ouf... j'ai cru que j'allais me prendre la camionnette! » alors qu'elle passait (dans la marche équipée) entre deux obstacles, une camionnette blanche et un horodateur (Figure 6).

\footnotetext{
${ }^{21}$ Je n'ai retenu pour cet article que les commentaires au sujet de la réactivation, mais l'entretien qui a eu lieu pendant et après l'utilisation du dispositif a eu principalement comme sujet le trajet des participantes et les relations qu'elles entretiennent avec leur quartier au prisme des choix effectués lors de ce trajet.
} 


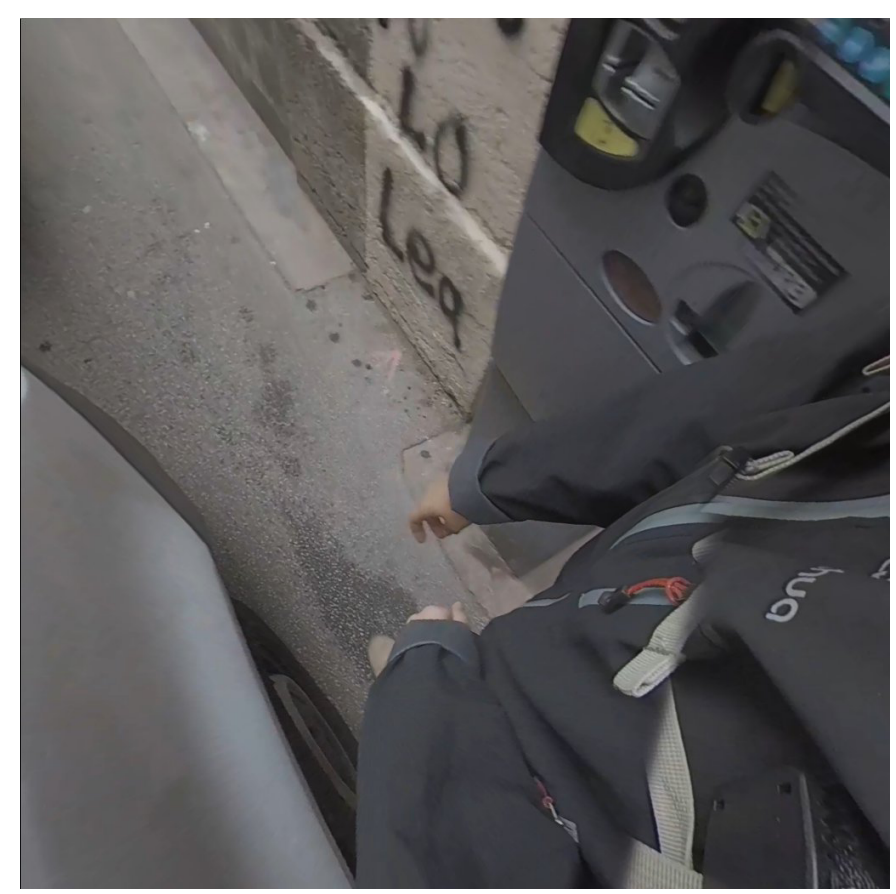

Figure 6 : Vue du casque de VR. Une participante passe entre deux obstacles. Crédits : Hugo Montero

Ce que j'interprète de cet instant-là, c' est justement le tissage dans lequel se trouve cette réactivation: la participante ne se souvient pas d'avoir heurté cette camionnette, mais pourtant lorsqu' elle visionne et entend ce moment au travers du casque cette possibilité reste incarnée, c'est-à-dire que c'est au sujet de son propre corps qu'elle perçoit la menace d'un accident. Elle est à la fois la spectatrice, mais aussi l'actrice de la situation. La distance qu'elle prend avec l'expérience est à même de la laisser confondre l'expérience présente avec celle éprouvée plus tôt dans la journée.

\section{CONCLUSION}

Les media que nous utilisons sur le terrain, qu'il s'agisse de l'écriture, de la photographie, du film, des enregistrements sonores ou de la vidéo, mettent en jeu différents rapports de distance. Ils font fluctuer notre manière de nous représenter le monde, en tant que chercheurs, mais aussi pour les participants qui dans les paradigmes récents sont invités à partager la production du terrain. Il est intéressant de comprendre les différences et similitudes qu'impliquent leurs utilisations sur le terrain. Pour répondre aux besoins de l'anthropologie du sensible, il est nécessaire de penser la place des media dans les expériences que vont partager avec nous les participants. Les troubles qui 
peuvent être reliés à l'utilisation de ces media, par les participants comme les chercheurs, sont à même de créer un retour réflexif sur leurs expériences. Pour prendre l'exemple de la vidéo,

«En ajoutant de la vidéo au processus de parler ou de raconter quelque chose [...] notre collaboration avec les participants implique non seulement leurs récits d'expériences sensorielles, mais aussi sa démonstration visuelle, l'exposition à des sons, des odeurs et des textures qui amènent l'ethnographe plus près du contexte sensoriel et pré réflexif de l'expérience. » (Pink, 2006 : 47)

La transformation de distance, ici explicitée par Sarah Pink, affine les rapports participants-terrain et chercheurs-terrain, leur permettant de partager l'expérience de la diffusion de cette vidéo ensemble, tout en échangeant à propos du sens à lui donner.

Le medium permet de créer un objet de discussion : il s'agit toujours de l'expérience de celui qui a produit la vidéo ou autre, mais il devient possible de partager ce qui a guidé sa production ou les sensations liées à sa réception. Dans les différentes méthodologies évoquées ici, il me semble central de considérer cette idée: utiliser un medium partagé entre chercheurs et participants (dans sa production et dans son interprétation), c'est créer un point duquel on peut partir ensemble vers une recherche de sens, qui passera nécessairement par des rapports de distance à l'objet.

L'utilisation d'images et d'enregistrements sonores à 360 degrés, diffusés dans un casque de réalité virtuelle et un casque audio, vise à multiplier les distances prises par les participants vis-à-vis de leur première expérience (ici la marche équipée) pour faire émerger un discours sensible - et non seulement visuel - et incarné à propos de leur expérience d'un trajet quotidien. C'est inviter les participants à poser une écoute, un regard, sur une expérience hybride, c'est-à-dire qui dénote avec ce qu'ils ont vécu, mais à laquelle ils peuvent raccrocher leurs souvenirs. Ce qui est discuté par les participants et les chercheurs lors de la réactivation présentée ici est un tissage entre la première expérience (la marche équipée) et la seconde (l'expérience réactivée).

Un tel dispositif présente bien sûr de nombreuses limites, et je souligne ici celle qui m'a parue la plus prégnante : sa lourdeur. Non seulement il demande aux personnes de s'habituer à une autre représentation du monde (et certaines ne s'y feront pas), mais il demande aussi un investissement temporel important, pour des raisons techniques comme pratiques. De plus, dans le cas de l'utilisation d'un casque de réalité virtuelle, j'ai pu constater que l'immersion dans l'expérience augmente avec le temps passé sous le casque. De la même manière, les chances de se sentir mal et de souffrir de cinétose augmentent avec la durée de l'expérience. Cette observation suggère qu'il est nécessaire de limiter l'expérience à de courts enregistrements ou bien de travailler avec des personnes habituées à ce type de visualisations, ce qui réduit les intérêts à mobiliser un tel appareillage. 
Malgré cela, le trouble sensoriel est bien présent et il invite d'une manière particulière - avec une certaine complicité - les participants à le partager et à le commenter avec les chercheurs. Par contre, au regard de tous les efforts consentis en ce sens, le discours sur l'expérience sort difficilement de l'oculocentrisme de notre perception, et les nombreux commentaires sont partagés à partir de ce que l'on voit plus que ce que l'on ressent. Sans doute faudrait-il envisager de croiser une telle méthodologie avec des méthodes d'entretiens spécifiquement pensées pour éliciter les sensations des participants, tels que les entretiens d'explicitation (Vermersh, 1994).

\section{BIBLIOGRAPHIE}

Sophie ACCOLAS, "Cinéma ethnographique expérimental. Références sommaires sur une pratique artistique et scientifique », Journal des anthropologues, num. 140-141, 2015, pp. 305-31.

Jean-François AUGOYARD, "L'entretien sur écoute réactivée », in Michèle GROSJEAN et Jean-Paul THIBAUD (dir.), L'espace urbain en méthodes, Marseille, Éditions Parenthèses, 2001, pp. 127-153.

Vincent BATTESTI, “"L'ambiance est bonne" » ou l'évanescent rapport aux paysages sonores au Caire. Invitation à une écoute participante et proposition d'une grille d'analyse », in Marie-Barbara LE GONIDEC et Joël CANDAU (dir.), Paysages sensoriels. Essai d'anthropologie de la construction et de la perception de l'environnement sonore, Éditions du CTHS, Aubervilliers, 2013, pp. 70-95.

Vincent BATTESTI et Nicolas PUIG, "The sound of society »: A method for investigating sound perception in Cairo", Marie-Luce GELARD (ed.), Dossier Contemporary French Sensory Ethnography, Senses and Society, 2016, pp. 298-319.

Howard Saul BECKER "Photography and Sociology", in Studies in Visual Communication, 1974, pp. 3-26.

Robert BONAMY, Le fond cinématographique, Le parti pris du cinéma, Paris, L'Harmattan, 2013.

Paul-Louis COLON et Joël CANDAU, (dir.) Ethnographier les sens, Paris, Éditions Pétra, 2014.

Marin DACOS, «Manifeste des Digital humanities », THATCamp. Non conférence sur les Digital Humanities, Paris, 2010, 26 mars 2011 [En ligne]. URL: https://tcp.hypotheses.org/318

Julien DELAS, "Cheminer "en aveugle" dans des lieux inconnus: perspectives réflexives sur la situation d'enquête », Specificites, 2015, pp.54-59.

Robert R DESJARLAIS, Sensory Biographies, University of California press, Berkeley, 2003.

James J. GIBSON, «Le système haptique », dans Nouvelles de danse : Vu du corps, traduction de Carole Guth, Contredanse, 2001, vol. 48-49, pp. 94-120. 
Anna GRIMSHAW et Amanda RAVETZ, Observational Cinema, Indiana University Press, Bloomington, 2009.

David HOWES, «Les techniques des sens », Anthropologie et Sociétés, 14(2), 1990, pp.99-115.

David HOWES, Empire of the Senses, Berg Publishers, Oxford, 2005.

Timothy INGOLD, Marcher avec les dragons, Zones Sensibles, Bruxelles, 2013.

Dominique KALIFA, « Préface », Hypothèses, vol. 15, no. 1, 2012, pp. 9-12.

Christian LALLIER, "L'observation filmante. Une catégorie de l'enquête ethnographique ", L'Homme. Revue française d'anthropologie, 198-199, 2011, pp.105130.

François LAPLANTINE, « Penser en images », Ethnologie francaise, 1/37, 2007, pp. 47-56.

David MACDOUGALL, The Corporeal Image: Film, Ethnography, and the Senses, Princeton University Press, Princeton, 2006.

Bronislaw MALINOWSKI, Journal d'ethnographe, Paris, Le Seuil, 1985 [1967].

Margaret MEAD et Gregory BATESON, "On the Use of the Camera in Anthropology", Anthropology of Media, Blackwell Publishing, Oxford, Askew, Wilk, 2002 [1977], pp.40-46.

Perle MØНL, «Médias sensoriels en anthropologie: exploration d'une complicité », Champ social, 2/15, 2015, pp.47-53.

Eadweard MUYBRIDGE, The human figure in motion, London, Chapman \& Hall, 1901.

Vincent O'BRIEN et al., "Embracing the Everyday: Reflections on Using Video and Photography in Health Research", Proceedings of the Workshop on Social Interaction and Mundane Technologies, SIMTECH 08, 2008.

Vincent O'BRIEN, Manpreet DHUFFAR et GRIFFITHS Mark D., "Collaborative Visual Ethnography: Practical Issues in Cross-Cultural Research", SAGE Research Methods Cases, London, SAGE Publications, 2014.

Gerald OSTER, "Auditory Beats In The Brain - Binaural Beats Meditation", in Scientific American, 229/4, 1973, pp.94-102.

Walter J. ONG, The Presence of the Word, New Haven, Yale University Press, 1967.

Sarah PINK, The Future of Visual Anthropology, Routledge, London and New York, 2006.

Raymond Murray SCHAFER, Le paysage sonore, Paris, J.-C. Lattés, 1979.

Michel SERRES, Les Cinq Sens, Grasset, Paris, 1985.

Jean-Fabien STECK, «Être sur le terrain, faire du terrain », Hypothèses, pp.75-84, 2012.

Pierre VERMERSCH, L'entretien d'explicitation, collection pédagogie, Paris, ESF, 1994.

Caroline WANG et Mary Ann BURRIS, "Empowerment through Photo Novella: Portraits of Participation", Health Education Quarterly, 21/2, 1994, pp.171-186. 
Caroline WANG et Mary Ann BURRIS, "Photovoice: Concept, Methodology, and Use for Participatory Needs Assessment", Health Education \& Behavior, 24/3, 1997, pp.369-387.

RÉSUMÉ : Cet article vise à approcher la manière dont les outils audiovisuels modifient le rapport qu'entretiennent les chercheurs et chercheuses au terrain et aux personnes participant à ce dernier. Au travers de la notion de distance, c'est-à-dire l'intervalle qui conditionne l'aller-retour entre ce dont nous faisons l'expérience, en tant que chercheurs, et ce que nous écrivons à propos de cette dernière, je souhaite mettre en évidence les différents aspects de notre expérience sensorielle. Entre trouble et immersion, j'utiliserai mes récents travaux, à l'aide de la "réalité virtuelle » pour exemplifier ce que permet le développement des humanités numériques dans le cadre d'une anthropologie du sensible.

MOTS-CLÉS : Anthropologie du sensible, réalité virtuelle, sensorialité, humanités numériques, anthropologie visuelle, videovoice, ethnographie expérimentale. 